\title{
Detection and Identification of a Substance with an Inhomogeneous Surface Using the Effective Time-Dependent THz Spectroscopy Method and Emission Frequency Up-Conversion
}

\author{
Vyacheslav A. Trofimov $^{1,2}$, Svetlana A. Varentsova ${ }^{2}$, Yongqiang Yang ${ }^{1}$ \\ ${ }^{1}$ South China University of Technology, Guangzhou, China, vatro@cs.msu.ru \\ ${ }^{2}$ M.V. Lomonosov Moscow State University, Moscow, Russian Federation
}

Currently, the $\mathrm{THz}$ radiation is actively applied for solving security and anti-terrorism problems [1], [2]. In most THz TDS setups, the substance identification occurs based on comparison of the absorption frequencies of a substance under investigation with a set of known absorption frequencies from a database (we call it as the standard THz TDS method). However, this technology has certain limitations.

It is well-known that surface roughness can lead to scattering and modulating of the spectrum, which complicates the identification [3], [4]. To overcome this problem, in [3] it was shown that by summing and averaging multiple measurements over a sample area the scattering effect can be effectively decreased. In [4] the authors proposed for the same purpose to increase the number of viewing angles. However, they can reliably identify only one absorption frequency with maximal spectral amplitude.

Unlike the methods mentioned above, in the discussed method we use only one $\mathrm{THz}$ signal reflected from the substance with inhomogeneous surface but measured in the long time interval duration 180-200 ps. This duration allows registering not only the main reflected $\mathrm{THz}$ pulse, but also several sub-pulses following it, which are due to the reflectance from the inner surfaces of the sample. These sub-pulses also contain information about spectral characteristics of the substance and can be used for the identification. In addition, we do not apply averaging of measurements over viewing angles and the sample area in order to reduce the scattering effects.

As an example of identification, we use the $\mathrm{THz}$ signal duration $180 \mathrm{ps}$ reflected from the PWM C4 explosive (90\% RDX, $10 \%$ plasticizer) with a rough surface grit 40 (signal PWM_40 for brevity). The signal contains the pronounced main pulse reflected from the outer surface of the sample, the first sub-pulse reflected from the inner surface, and the sub-pulses with significantly less amplitude due to multiple reflections from the inner surfaces. The Fourier spectrum of the PWM_40 main pulse is shown in Fig. 1 (a) together with that of the smooth PWM for comparison.

The spectral properties of the PWM_40 main pulse are distorted by the influence of the inhomogeneous surface (a) so that the standard THz TDS method is inefficient for identification. In the PWM_40 spectrum (a) there is a single minimum at the frequency $v=0.88 \mathrm{THz}$ in the frequency range $v=[0,1.8]$ $\mathrm{THz}$, which is close to RDX absorption frequency $v=0.82 \mathrm{THz}$. In the frequency range $v=[1.8,4.0] \mathrm{THz}$ there are minima at the frequencies $v=1.92,2.24,3.0$ $\mathrm{THz}$, which are close or equal to the RDX absorption frequencies $v=1.95,2.19,3.0 \mathrm{THz}$ [2].

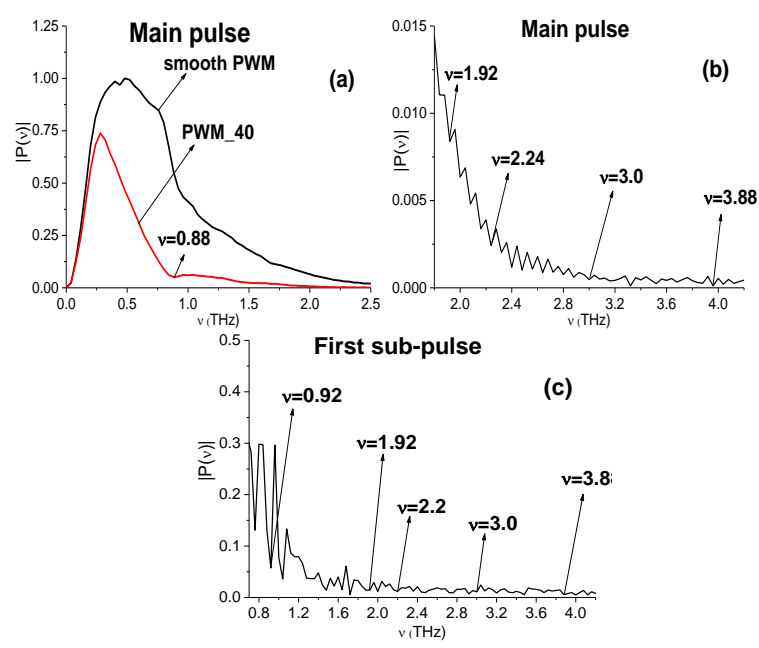

Fig. 1. Fourier spectrum of the main pulse of the PWM_40 and smooth PWM signals in the frequency ranges $v=[0.0$, 2.5] THz (a), [1.8, 4.2] THz (b), spectrum of the PWM_40 first sub-pulse in the frequency range $v=[0.8,4.2] \mathrm{THz}(\mathrm{c})$.

The spectrum of the PWM_40 first sub-pulse contains minima at the frequencies $v=0.92,1.92,2.2$. 3.0 THz (c), that are close to the absorption frequencies of explosive RDX $v=0.82,1.95,2.19,3.0 \mathrm{THz}$ ([2]). And the spectrum of the remote part of the PWM_40 signal corresponding to the time interval $\mathrm{t}=[70,170] \mathrm{ps}$, contains minima at the frequencies $v=$ $0.9,1.96,2.2,3.01 \mathrm{THz}$, which are close to RDX absorption frequencies mentioned above (not shown). Therefore, the sub-pulses also contain information about the substance spectral properties and can be used for identification.

In the current report, the identification is based on the method of spectral dynamics analysis (SDAmethod) together with several integral correlation criteria (ICC) [5]. We compare the dynamics of spectral intensity of a substance under analysis with the corresponding dynamics of a standard substance from database at chosen frequencies. To increase the reliability and effectiveness of the identification, we propose to use several ICC's simultaneously in different time intervals, which contain both the main pulse of the reflected $\mathrm{THz}$ signal and the following sub-pulses.

In Fig. 2 the evolution of the ICC $C W 1_{p, P}$ [5] is calculated for the frequencies $v=0.82 \mathrm{THz}$ (a), (c) and 
$v=3.0 \mathrm{THz}(\mathrm{b}),(\mathrm{d})$ in the time intervals, which contain the main pulse (a), (b) and the first sub-pulse (c), (d) of the PWM_40 signal. In all cases (a)-(d) the frequencies $v=0.82,3.0 \mathrm{THz}$ are detected as RDX absorption frequencies. The same result is valid for the time interval $t=[70,170] \mathrm{ps}$, containing two other subpulses with less amplitude. The ICC $C_{p, P}$ [5] confirms these results.
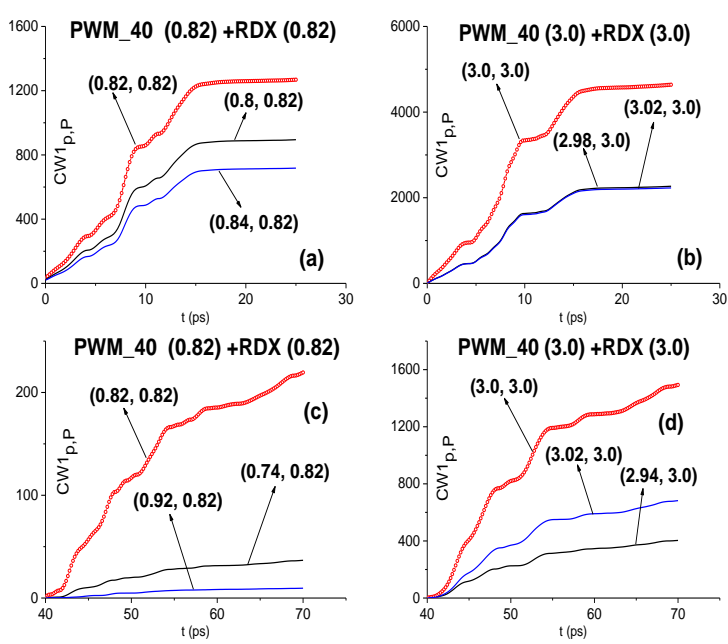

Fig. 2. ICC $C W 1_{p, P}\left(t_{n}\right)$ detecting frequencies $v=0.82 \mathrm{THz}$ (a), (c), $3.0 \mathrm{THz}$ (b), (d) in the time intervals $\mathrm{t}=[0,30] \mathrm{ps}$ (a), (b), $[40,70]$ ps (c), (d).

Another promising approach for the substance detection and identification is related to the frequency up-conversion based on a broadband $\mathrm{THz}$ pulse. The $\mathrm{THz}$ pulse induces high energy level excitation of molecules due to essential non-stationary medium response. This leads to the substance emission at frequencies, which are absent in the incident pulse spectrum. Earlier the frequency up-conversion and the appearance of the doubled current frequency in the $\mathrm{THz}$ signals transmitted through or reflected from the medium without and with covering were demonstrated using a mathematical modelling in [6]. The mathematical model is based on the 1D Maxwell-Bloch equations and density matrix formalism. In the current report, we investigate the possible manifestation of the frequency-up conversion in the $\mathrm{THz}$ signal PWM_40 reflected from the substance with a rough surface.

Note that the spectra of the PWM_40 main pulse Fig. 1 (b) and the first sub-pulse Fig. 1 (c) contain minima at the frequency $v=3.88 \mathrm{THz}$, which is close to the doubled frequency $v=1.92 \cdot 2 \mathrm{THz}$. The same is observed in the PWM_40 spectrum corresponding to the time interval $\mathrm{t}=[70,170] \mathrm{ps}$.

In Fig. 3 the evolution of the ICC $C W 1_{p, P}$ calculated for the frequency pair $v=(3.88,1.92) \mathrm{THz}$ is shown in the time intervals containing the PWM_40 main pulse (a) and the first sub-pulse (b). In both cases, the frequency $v=3.88 \mathrm{THz}$ is detected as the doubled RDX frequency in the reflected signal PWM_40. The ICC $C_{p, P}$ [5] also confirms these results.
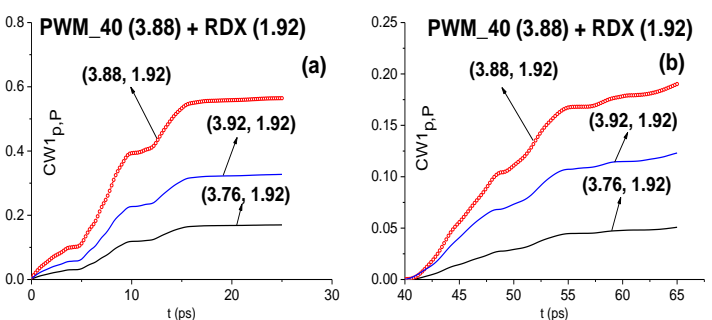

Fig. 3. ICC $C W 1_{p, P}$ calculated for the PWM_40 signal at the doubled frequency $v=3.88 \mathrm{THz}$ and the standard signal RDX_Air at the frequency $v=1.92 \mathrm{THz}$ in the time intervals $\mathrm{t}=[0,25] \mathrm{ps}(\mathrm{a}),[40,65] \mathrm{ps}(\mathrm{b})$.

We propose an effective time-dependent $\mathrm{THz}$ spectroscopy method, which allows detecting and identifying a substance with inhomogeneous surface using only one long-duration reflected $\mathrm{THz}$ signal without averaging of the measured $\mathrm{THz}$ signals over the viewing angles and scanning over the surface area. For successful analysis and identification, the registered $\mathrm{THz}$ signal must contain both the main reflected $\mathrm{THz}$ pulse and several sub-pulses. This feature of the method significantly increases the signal processing speed and allows us to use it in real time.

The possibility for the substance detection and identification using the doubled current frequency is shown with the help of the SDA-method and integral correlation criteria. This result is important for practice, because the frequencies belonging to the high frequency range can be used for the substance detection and identification even in the case when the lower substance absorption frequencies are suppressed by covering or noise influence.

The $\mathrm{THz}$ signals reflected from the PWM C4 sample were measured in the Military University of Technology (Warsaw, Poland); THz signal transmitted through the RDX sample - in the Center for Terahertz Research, Rensselaer Polytechnic Institute (NY, USA).

\section{References}

1. Leahy-Hoppa, M. R, Fitch, M. J., Zheng, X., et all. Wideband terahertz spectroscopy of explosives // Chem. Phys. Lett. 2007. V. 434. P. 227-230.

2. Liu, H. B., Zhong, H., Karpowicz, N., et all. THz spectroscopy and imaging for defense and security applications // Proc. IEEE. 2007. V. 95, No. 8, P. 1514-1527.

3. Shen, Y. C., Taday, P. F., Pepper, M. Elimination of scattering effects in spectral measurement of granulated materials using terahertz pulsed spectroscopy // Appl. Phys. Lett. 2008. V. 92, No. 5. Paper 051103.

4. Henry, S., Kniffin, G., Schecklman, S., et all. Measurement and modeling of rough surface effects on terahertz spectroscopy and imaging // Proc. SPIE. 2010. V. 7601. Paper 760108.

5. Trofimov, V. A., Varentsova, S. A. Detection and identification of drugs under real conditions by using noisy terahertz broadband pulse // Appl. Opt. 2016. V. 55, No. 33. P. 9605-9618.

6. Trofimov, V. A., Zakharova, I. G., Zagursky, D. Yu., Varentsova, $S$. A. New approach for detection and identification of substances using THz TDS // Proc. SPIE. 2017. V. 10441. Paper 1044107. 